\title{
Decoherence of localized spins interacting via RKKY interaction
}

\author{
Yoshiaki Rikitake and Hiroshi Imamura \\ CREST and Graduate School of Engineering, Tohoku University, Sendai 980-8579, Japan
}

\begin{abstract}
We theoretically study decoherence of two localized spins interacting via the RKKY interaction in one-, two-, and three-dimensional electron gas. We derive the kinetic equation for the reduced density matrix of the localized spins and show that energy relaxation caused by singlet-triplet transition is suppressed when the RKKY interaction is ferromagnetic. We also estimate the decoherence time of the system consisting of two quantum dots embedded in a two dimensional electron gas.

PACS numbers: 03.65.Yz, 75.75.+a, 75.50.-y
\end{abstract}

Quantum computation and quantum information are emerging research fields of physics, technology and information sciences [1]. The elementary units in most quantum computation and quantum information schemes are a quantum bit and a quantum gate. Because of its scalability and relatively long coherence time 2], solid state device with localized spins is considered as one of the promising candidates for these quantum devices 3 . Kane 4] proposed the system of nuclear spins of phosphorus donors in a silicon heterostructure with direct exchange interaction between electrons localized at the donors. Mozyrsky et al. [5, [6] proposed the qubits of nuclear spins in quantum-Hall system in which the gate operation is realized by the indirect exchange interaction via virtually excited spin waves. Recently Craig et al. [7, 8] observed the RKKY [9] coupling of semiconductor quantum dots, which proved that the RKKY interaction can be used as a quantum gate consisting of localized spins in semiconductor quantum dots. Several theoretical papers studying the RKKY interaction in such semiconductor nanostructures have been published 10, 11, 12, 13, 14, 15].

One of the major obstacle to realizing quantum computation is decoherence 16, 17]. Since the RKKY interaction is mediated by the electron gas, the particle-hole excitations act as an environment (Fermion bath) 18, 19, 20, 21]. It is therefore important to clarify the effects of the Fermion bath on the dynamics of the qubits consisting of the localized spins.

In this paper, we study the dynamics of the localized spins interacting via the RKKY interaction by using the kinetic equation of the reduced density matrix. We find the term intrinsic to the RKKY interaction appears in the kinetic equation, which is an oscillating function of the distance between the localized spins with the same period as the RKKY interaction. We show that the energy relaxation due to the singlet-triplet transition is strongly suppressed by this term. We also discuss the physical realization of a quantum gate using the RKKY interaction and estimate the decoherence time of the system consisting of two quantum dots embedded in a two dimensional electron gas(2DEG).

We consider the system consisting of two localized spins embedded in a one-, two-, or three-dimensional electron gas. The Hamiltonian, $H=H_{S}+H_{\mathrm{c}}+H_{\text {int }}$, com- prises the localized spin part, $H_{\mathrm{S}}$, the conduction electron part, $H_{\mathrm{c}}$, and the $s$ - $d$ interaction, $H_{\text {int }}$. We assume that there is no external magnetic field and we set $H_{\mathrm{S}}=0$. In the second quantization representation, $H_{\mathrm{c}}$ and $H_{\text {int }}$ are expressed as

$$
\begin{aligned}
H_{\mathrm{c}} & =\sum_{\boldsymbol{k} \sigma} \varepsilon_{k} c_{\boldsymbol{k} \sigma}^{\dagger} c_{\boldsymbol{k} \sigma}, \\
H_{\mathrm{int}} & =\frac{J}{2 V} \sum_{p=1,2} \sum_{\boldsymbol{k}, \boldsymbol{k}^{\prime}} e^{i\left(\boldsymbol{k}-\boldsymbol{k}^{\prime}\right) \cdot \boldsymbol{R}_{p}}\left\{S_{p}^{-} c_{\boldsymbol{k}^{\prime} \uparrow}^{\dagger} c_{\boldsymbol{k} \downarrow}\right. \\
& \left.+S_{p}^{+} c_{\boldsymbol{k}^{\prime} \downarrow}^{\dagger} c_{\boldsymbol{k} \uparrow}+S_{p}^{z}\left(c_{\boldsymbol{k}^{\prime} \uparrow}^{\dagger} c_{\boldsymbol{k} \uparrow}-c_{\boldsymbol{k}^{\prime} \downarrow}^{\dagger} c_{\boldsymbol{k} \downarrow}\right)\right\},
\end{aligned}
$$

where $c_{\boldsymbol{k} \sigma}^{\dagger}$ and $c_{\boldsymbol{k} \sigma}$ are creation and annihilation operators of an electron with wavenumber vector $\boldsymbol{k}$ and spin $\sigma$. Here $\varepsilon_{k}$ is an energy of conduction electrons, $J$ is a coupling constant of $s$-d interaction, and $\boldsymbol{R}_{p}(p=1,2)$ represents the position of the localized spin $\boldsymbol{S}_{p}$. We assume that the coupling constant $J$ is so small that we can treat $H_{\text {int }}$ as a perturbation on $H_{\mathrm{c}}$.

The dynamics of the two localized spins is described by the reduced density matrix $\rho(t)=\operatorname{tr}_{\mathrm{c}}\left\{\rho_{\text {tot }}(t)\right\}$, where $\rho_{\text {tot }}$ is the density matrix for the total system and $\operatorname{tr}_{\mathrm{c}}$ means trace over the degrees of freedom of the conduction electrons. The reduced density matrix $\rho(t)$ obeys the following kinetic equation [22, 23, 24, 25]:

$$
\frac{d}{d t} \rho(t)=-\frac{i}{\hbar}\left[H_{S}, \rho(t)\right]+\int_{0}^{t} d t^{\prime} \Sigma\left(t-t^{\prime}\right) \rho\left(t^{\prime}\right) .
$$

The self-energy $\Sigma\left(t-t^{\prime}\right)$ is a super-operator acting on $\rho(t)$. We assume that at the initial time $t=0$ the system of the localized spins and the system of the conduction electrons are decoupled, and the conduction electrons are in thermal equilibrium: $\rho_{\text {tot }}(0)=\rho(0) \otimes \rho_{\mathrm{c}}^{e q}$, where $\rho_{\mathrm{c}}^{e q}=$ $e^{-\left(H_{\mathrm{c}}-\mu N\right) / k_{B} T} / \operatorname{tr}_{\mathrm{c}} e^{-\left(H_{\mathrm{c}}-\mu N\right) / k_{B} T}$.

We carry out a second-order perturbative calculation for the self-energy $\Sigma\left(t-t^{\prime}\right)$ in Eq.(3). We assume that the reduced density matrix $\rho(t)$ varies slowly compared to the lifetime of particle-hole excitations, and we make the Markov approximation in Eq.(3). A straight forward calculation gives

$$
\frac{d}{d t} \rho(t)=\left(\mathcal{H}_{\mathrm{RKKY}}+\sum_{p=1,2} \mathcal{D}_{p}+\mathcal{D}_{\mathrm{ex}}\right) \rho(t),
$$




\begin{tabular}{c|c|c|c|c}
\hline$d$ & $\alpha_{d}$ & $\beta_{d}$ & $F_{d}(x)$ & $G_{d}(x)$ \\
\hline \hline 1 & $\frac{m^{2} J^{2}}{2 \pi \hbar^{4} k_{F}^{2}}$ & $2 \operatorname{si}(\pi)=5.62 \times 10^{-1}$ & $\frac{\operatorname{si}(x)}{\operatorname{si}(\pi)}$ & $\frac{1+\cos x}{2}$ \\
\hline 2 & $\frac{m^{2} J^{2}}{32 \pi^{2} \hbar^{4}}$ & $\frac{8}{\pi} J_{1}\left(z_{0}\right) N_{1}\left(z_{0}\right)=1.10 \times 10^{-1}$ & $\frac{J_{0}(x / 2) N_{0}(x / 2)+J_{1}(x / 2) N_{1}(x / 2)}{J_{1}\left(z_{0}\right) N_{1}\left(z_{0}\right)}$ & $J_{0}^{2}(x / 2)$ \\
\hline 3 & $\frac{m^{2} J^{2} k_{F}^{2}}{16 \pi^{3} \hbar^{4}}$ & $\frac{1}{\pi^{3}}=3.23 \times 10^{-2}$ & $(2 \pi)^{3} \frac{x \cos x-\sin x}{x^{4}}$ & $2 \frac{1-\cos x}{x^{2}}$ \\
\hline
\end{tabular}

TABLE I: The coefficients $\alpha_{d}$ and $\beta_{d}$, and the range functions $F_{d}(x)$ and $G_{d}(x)$ for $d$-dimensional electron gas $(d=1,2,3)$. $\operatorname{si}(x) \equiv-\int_{x}^{\infty} d t \sin t / t$ is the sine integral function. $J_{n}$ is the Bessel function and $N_{n}$ is the Neumann function of order $n$. $z_{0}=2.40$ is the first zero-point of $J_{0}$.

where $\mathcal{H}_{\mathrm{RKKY}}, \mathcal{D}_{p}(p=1,2)$, and $\mathcal{D}_{\text {ex }}$ are the superoperators defined as follows.

The first term of r.h.s. in Eq.(4) is defined as

$$
\mathcal{H}_{\mathrm{RKKY}} \rho(t)=-i \frac{J_{\mathrm{RKKY}}}{\hbar}\left[\boldsymbol{S}_{1} \cdot \boldsymbol{S}_{2}, \rho(t)\right]
$$

which represents the coherent time evolution of the two localized spins interacting via the RKKY interaction 9 , 26, 27, 28]. The effective coupling constant $J_{\mathrm{RKKY}}$, for $d$-dimensional system $(d=1,2,3)$ is given by $J_{\mathrm{RKKY}}=$ $\alpha_{d} \beta_{d} E_{F} F_{d}\left(2 k_{F} R\right)$, where $R=\left|\boldsymbol{R}_{1}-\boldsymbol{R}_{2}\right|$ is the distance between two localized spins, $E_{F}$ is the Fermi energy, and $k_{F}$ is the Fermi wavenumber. Here $\alpha_{d}, \beta_{d}$, and the range function $F_{d}(x)$ are given in Table I The range functions $F_{d}\left(2 k_{F} R\right)$ for $d=1,2$, and 3 are plotted by dotted lines in Figs प(a), (b), and (c), respectively. The RKKY interaction is produced by virtually excited quantum states within a wide energy window from the bottom of energy band to the Fermi surface. Therefore, the strength of the RKKY interaction, $J_{\mathrm{RKKY}}$, is proportional to the Fermi energy $E_{F}$ and is oscillating function of $R$ with the period of half of the Fermi wavelength, $\pi / k_{F}$.

The second term of r.h.s. in Eq.(4) is defined as

$$
\begin{aligned}
\sum_{p=1,2} \mathcal{D}_{p} \rho(t) & =-\frac{\gamma}{\hbar} \sum_{p=1,2}\left(\frac{3}{2} \rho(t)-\left(S_{p}^{+} \rho(t) S_{p}^{-}\right.\right. \\
& \left.\left.+S_{p}^{-} \rho(t) S_{p}^{+}+2 S_{p}^{z} \rho(t) S_{p}^{z}\right)\right)
\end{aligned}
$$

where the coefficient $\gamma$ is expressed as $\gamma=\alpha_{d} k_{B} T$. This term describes the usual decoherence of a localized spin $\boldsymbol{S}_{p}$ interacting with the Fermion bath, which is known as the Korringa relaxation 29]. Since thermally excited particle-hole pairs cause the Korringa relaxation, $\gamma$ is proportional to the temperature $T$. One can easily show that this term causes energy relaxation due to transition between singlet and triplet states and dephasing due to transition among three degenerate triplet states.

The last term of r.h.s. in Eq.(44), which is intrinsic to the system with the RKKY interaction, is defined as

$$
\begin{aligned}
\mathcal{D}_{\text {ex }} \rho(t) & =-\frac{2 \gamma_{\mathrm{ex}}}{\hbar}\left(\left\{\boldsymbol{S}_{1} \cdot \boldsymbol{S}_{2}, \rho(t)\right\}-\frac{1}{2}\left(S_{1}^{+} \rho(t) S_{2}^{-}\right.\right. \\
& \left.\left.+S_{1}^{-} \rho(t) S_{2}^{+}+2 S_{1}^{z} \rho(t) S_{2}^{z}+(\operatorname{spin} 1 \leftrightarrow 2)\right)\right) .
\end{aligned}
$$

This term exists only when the two localized spins interact with each other via conduction electrons. The coefficient $\gamma_{\mathrm{ex}}$ is expressed as $\gamma_{\mathrm{ex}}=\alpha_{d} k_{B} T G_{d}\left(2 k_{F} R\right)$. The Range function $G_{d}\left(2 k_{F} R\right)$ is given in table【 and plotted by solid lines in Figs 1(a),(b), and (c). As we shall show later, this term suppresses the energy relaxation caused by singlet-triplet transitions due to the term $\mathcal{D}_{p}$. The origin of this term is interference among thermally exited particle-hole pairs near the Fermi surface. Therefore, the range function $G_{d}\left(2 k_{F} R\right)$ oscillates with the same period as $F_{d}\left(2 k_{F} R\right)$. Reflecting the difference in physical mechanisms behind them, the amplitude of $G_{d}\left(2 k_{F} R\right)$ decays as $1 / R^{d-1}$ while that of $F_{d}\left(2 k_{F} R\right)$ decays as slowly as $1 / R^{d}$. Especially for the one-dimensional electron gas, the amplitude of the oscillation of $G_{d}\left(2 k_{F} R\right)$ does not decay as $R$ increases.

Next, in order to capture the physical meaning of the term $\mathcal{D}_{\text {ex }} \rho(t)$, we examine the time evolution of the expectation value $\left\langle\boldsymbol{S}_{1} \cdot \boldsymbol{S}_{2}\right\rangle=\operatorname{tr}\left\{\rho(t) \boldsymbol{S}_{1} \cdot \boldsymbol{S}_{2}\right\}$, which is proportional to the energy expectation value of the RKKY interaction. By using Eqs.(4)-(6), we can show that it decays exponentially as $\left\langle\boldsymbol{S}_{1} \cdot \boldsymbol{S}_{2}\right\rangle \propto \exp \left(-4\left(\gamma-\gamma_{\text {ex }}\right) t / \hbar\right)$. Then the characteristic time of energy relaxation of the RKKY interaction is given by $\tau_{\mathrm{RKKY}}=\hbar / 4\left(\gamma-\gamma_{\mathrm{ex}}\right)$. Since the term $\mathcal{D}_{\text {ex }} \rho(t)$ is proportional to $\gamma_{\text {ex }}$, we can say that the term $\mathcal{D}_{\text {ex }} \rho(t)$ tends to suppress energy relaxation. Note that $\gamma_{\text {ex }}$ does not exceeds $\gamma$. Especially when $\gamma_{\mathrm{ex}}=\gamma$, the singlet-triplet transitions due to the term $\mathcal{D}_{p}$ does not occur and energy relaxation of the RKKY interaction is completely suppressed. However, the transitions among the triplet states are not suppressed and dephasing rate remains finite even if $\gamma_{\mathrm{ex}}=\gamma$. As shown in Figs 1(a), (b), and (c), the range function $G_{d}\left(2 k_{F} R\right)$ takes its maximal values when the $F_{d}\left(2 k_{F} R\right)$ is negative. Consequently the energy relaxation of the RKKY interaction is suppressed when the RKKY interaction forms 


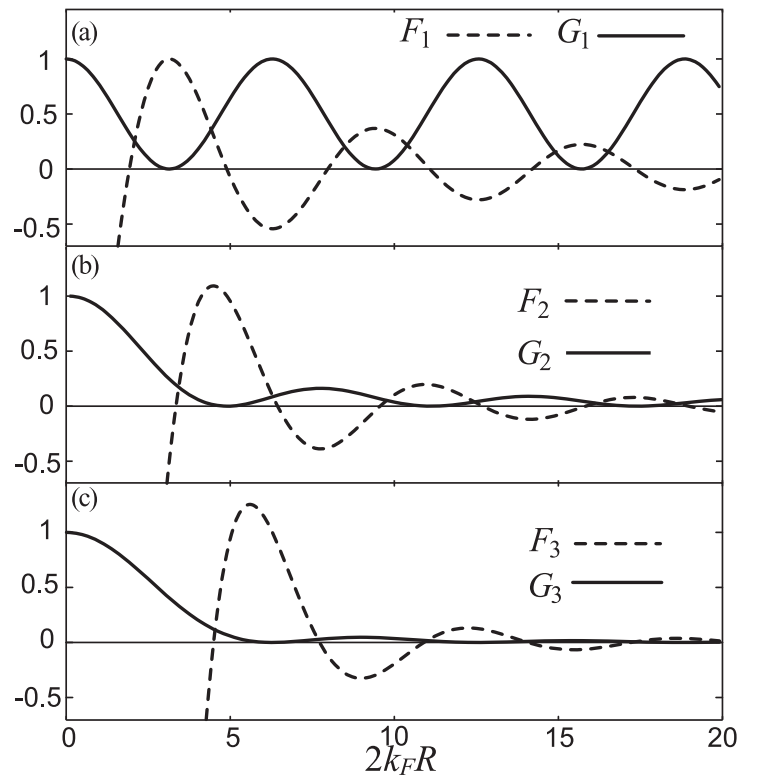

FIG. 1: The range functions for one-dimensional system $F_{1}\left(2 k_{F} R\right)$ and $G_{1}\left(2 k_{F} R\right)$ are plotted as a function of $2 k_{F} R$ in Panel (a). Panels (b) and (c) are the same plot for twoand three-dimensional systems, respectively.

ferromagnetic coupling $\left(J_{\mathrm{RKKY}}<0\right)$.

Figure 2(a) shows the time evolution of the diagonal element $\rho_{\uparrow \downarrow, \uparrow \downarrow}(t)$ of the reduced density matrix. The initial state is taken to be $|\uparrow \downarrow\rangle$ and the time scale is normalized by $h / J_{\text {RKKY }}$. The diagonal element $\rho_{\uparrow \downarrow, \uparrow \downarrow}(t)$ represents the occupation probability of the state $|\uparrow \downarrow\rangle$. Without decoherence, $\gamma=\gamma_{\mathrm{ex}}=0$, the quantum state of the two-spin system oscillates between $|\uparrow \downarrow\rangle$ and $|\downarrow \uparrow\rangle$ coherently and $\rho_{\uparrow \downarrow, \uparrow \downarrow}(t)$ shows a clear oscillation like a trigonometric function as indicated by the thin solid line in Fig 2(a). In the case of $\gamma=0.05 J_{\mathrm{RKKY}}$ and $\gamma_{\mathrm{ex}}=0$, where each spins decohere independently, $\rho_{\uparrow \downarrow, \uparrow \downarrow}(t)$ shows damped oscillation due to $\mathcal{D}_{p}$ (thick solid line). The dotted, dashed, and dot-dashed lines in Fig. 2(a) show the results for $\gamma_{\mathrm{ex}}=0.025,0.045$, and $0.05 J_{\mathrm{RKKY}}$, respectively. The value of $\gamma_{\text {ex }}$ can be controlled by changing the distance between localized spins, $R$. One can see that the oscillation amplitude of $\rho_{\uparrow \downarrow, \uparrow \downarrow}(t)$ decays more slowly as $\gamma_{\text {ex }}$ increases since the energy relaxation of the RKKY interaction is suppressed by $\gamma_{\mathrm{ex}}$. Although energy relaxation of the RKKY interaction is completely suppressed when $\gamma_{\mathrm{ex}}=\gamma$, dephasing due to the transition among the triplet states occurs as indicated by the dot-dashed line in Fig. 2(a).

In Fig. 2(b) we plot the off-diagonal element $\rho_{\uparrow \downarrow, \downarrow \uparrow}(t)$ with the initial state $(|\uparrow \downarrow\rangle-|\downarrow \uparrow\rangle) / \sqrt{2}$ (the singlet state, i.e., the maximally entangled state of the localized spins). Because the singlet state is one of the energy eigenstates of the RKKY interaction, $\rho_{\uparrow \downarrow, \downarrow \uparrow}(t)$ is the conserved quantity when $\gamma=\gamma_{\mathrm{ex}}=0$. If $\gamma$ takes a finite value, the singlet state changes to the mixed state and $\rho_{\uparrow \downarrow, \downarrow \uparrow}(t)$ decays

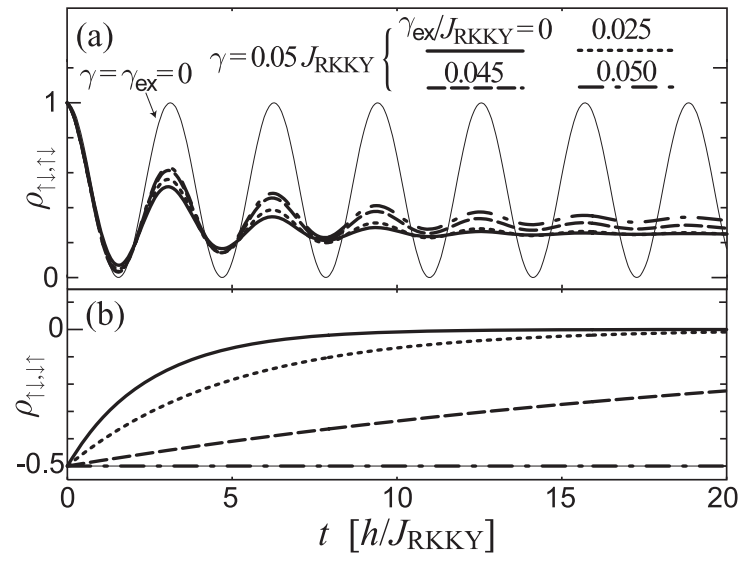

FIG. 2: (a) The time evolution of $\rho_{\uparrow \downarrow, \uparrow \downarrow}(t)$. The initial state is taken to be $|\uparrow \downarrow\rangle$ and the time is normalized by $h / J_{\text {RKKY }}$. (b) The same plot for $\rho_{\uparrow \downarrow, \downarrow \uparrow}(t)$ with the initial state $(|\uparrow \downarrow\rangle-|\downarrow \uparrow\rangle) / \sqrt{2}$. For both panels, thin solid line represents the result for $\gamma=\gamma_{\mathrm{ex}}=0$ while all the other lines are for $\gamma=0.05 J_{\mathrm{RKKY}}$. The parameter $\gamma_{\mathrm{ex}}$ is taken to be $0,0.025,0.045$, and $0.05 J_{\mathrm{RKKY}}$ for the thick solid, dotted, dashed, dot-dashed lines, respectively.

exponentially to zero. As shown in Fig[2 (b), $\rho_{\uparrow \downarrow, \downarrow \uparrow}(t)$ decays more slowly as $\gamma_{\text {ex }}$ increases, which means that $\gamma_{\text {ex }}$ suppresses decoherence of the singlet state. Especially in the case of $\gamma=\gamma_{\mathrm{ex}}$, the singlet state remains as it is forever and the off-diagonal element $\rho_{\uparrow \downarrow, \downarrow \uparrow}(t)$ never decays although the localized spins always interact with the Fermion bath of conduction electrons. One can easily show that for the singlet state the contributions of $\mathcal{D}_{\text {ex }} \rho(t)$ and $\mathcal{D}_{p} \rho(t)$ in Eq. (4) cancel out when $\gamma=\gamma_{\text {ex }}$.

Finally, we would like to discuss the physical realization of a quantum gate using the RKKY interaction and estimate the decoherence time. Let us consider the system consisting of two quantum dots embedded in a 2DEG of GaAs/AlGaAs heterostructure. We assume that the electron density of the 2DEG is $n^{2 \mathrm{D}}=7.3 \times 10^{11} \mathrm{~cm}^{-2}$, the Fermi energy and the Fermi wavelength are $E_{F} \sim$ $26 \mathrm{meV}$ and $2 \pi / k_{F} \sim 29.4 \mathrm{~nm}$. We also assume that the charging energy of the quantum dot is $U=1.9 \mathrm{meV}$, the Lorentzian broadening of the localized-state energy with full width at half maximum (FWHM) is $\Gamma=295 \mu \mathrm{eV}$, and the temperature is $T=100 \mathrm{mK}$ 30, 31]. Each quantum dot contains a single localized spin which acts as a qubit. The coupling between localized spins, i.e., the quantum gate operation is controlled by applying a gate voltage to $2 \mathrm{DEG}$. For example the $\sqrt{\mathrm{SWAP}}$ gate operation [1, 32], which is known as a universal 2-bit quantum gate operation, can be carried out if strength of the RKKY interaction $J_{\mathrm{RKKY}}$ is controlled such that $\int d t J_{\mathrm{RKKY}}(t) / \hbar=\pi / 2$. We suppose that the inter dot distance is $R=\tilde{z}_{2} / k_{F} \sim 11 \mathrm{~nm}\left(\tilde{z}_{2}=2.40\right.$ is the first zeropoint of the Bessel function $J_{0}$ ), that is, $\gamma_{\mathrm{ex}}=0$ and the energy relaxation is not suppressed. The strength of the RKKY interaction takes $J_{\text {RKKY }} \sim 3.4 \mu \mathrm{eV}$ and the oper- 
ation time defined as $\tau_{\mathrm{op}} \equiv \pi \hbar / 2 J_{\mathrm{RKKY}}$ is $\sim 0.3 \mathrm{~ns}$. Since $\gamma_{\text {ex }}$ is assumed to be zero, the decoherence time is determined only by $\gamma$ and is estimated as $\tau_{\mathrm{dec}} \equiv \hbar / \gamma \sim 62 \mathrm{~ns}$. Therefore, about 200 times coherent $\sqrt{\text { SWAP }}$ operation can be achieved.

The decoherence of a localized spin due to phonon scattering is suppressed in a small quantum dot and long coherence time (order of millisecond) of a single spin was observed in self-assembled semiconductor quantum dots 2]. Therefore, the decoherence due to the Fermion bath of conduction electrons is dominant in this system.

More coherent quantum gate operation is available if we locate the quantum dots very close to each other $\left(k_{F} R \ll 1\right)$. In this case the value of $\gamma_{\text {ex }}$ approaches $\gamma$ and therefore we can take an advantage of the suppression of the energy relaxation. Furthermore we can also obtain very strong ferromagnetic RKKY interaction[11]. Experimentally quantum dot arrays with $3-10 \mathrm{~nm}$ diameter quantum dots have already been fabricated [33, 34, 35]. We believe that the system we con- sider can be realized by using such quantum dot arrays with a few nm inter dot distance.

In conclusion, we have derived the kinetic equation for the system of two localized spins embedded in an electron gas and have shown that particle-hole excitations in the electron gas cause not only the RKKY interaction but also decoherence of the two-spin system. We also show that the strength of decoherence as well as the RKKY interaction strongly depends on the distance between two spins, and energy relaxation due to singlet-triplet transition is suppressed when the RKKY interaction is ferromagnetic $\left(J_{\mathrm{RKKY}}<0\right)$. We also estimate the decoherence time of the system consisting of two quantum dots embedded in a two dimensional electron gas(2DEG) to be $\tau_{\text {dec }} \sim 60 \mathrm{~ns}$ within which $\sim 200$ coherent $\sqrt{\text { SWAP }}$ operations can be achieved.

We acknowledge $\mathrm{H}$. Ebisawa for critically reading the manuscript. This work was supported by CREST, MEXT.KAKENHI(No. 14076204 and No. 16710061), NAREGI Nanoscience Project, and NEDO Grant.
[1] M. A. Nielsen and I. L. Chuang, Quantum Computation and Quantum Information (CAMBRIDGE UNIVERSITY PRESS, UK, 2000).

[2] M. Kroutvar, Y. Ducommun, D. Heiss, M. Bichler, D. Schuh, G. Abstreiter, and J. J. Finley, Nature 432, 81 (2004).

[3] D.D.Awshalom, Semiconductor Spintronics and Quantum Computation (Springer, Berlin, 2002).

[4] B. Kane, Nature 393, 133 (1998).

[5] D. Mozyrsky, V. Privman, and M.L. Glasser, Phys. Rev. Lett. 86, 5112 (2001).

[6] D. Mozyrsky, V. Privman, and I.D. Vagner, Phys. Rev. B 63, 085313 (2001).

[7] N. J. Craig, J. M. Taylor, E. A. Lester, C. M. Marcus, M. P. Hanson, and A. C. Gossard, Science 304, 565 (2004).

[8] L. I. Glazman and R. C. Ashoori, Science 304, 524 (2004).

[9] C. Kittel, Solid State Physics, vol. 22 (Academic Press, New York, 1968).

[10] C. Piermarocchi, P. Chen, L. J. Sham, and D. G. Steel, Phys. Rev. Lett. 89, 167402 (2002).

[11] H. Tamura, K. Shiraishi, and H. Takayanagi, Jpn. J. Appl. Phys. Part 2 - Lett. Express Lett. 43, L691 (2004).

[12] H. Imamura, P. Bruno, and Y. Utsumi, Phys. Rev. B 69, 121303(R) (2004).

[13] Y. Utsumi, J. Martinek, P. Bruno, and H. Imamura, Phys. Rev. B 69, 155320 (2004).

[14] S. Sun, S. Cheng, and H. Lin, Appl. Phys. Lett. 84, 2862 (2004).

[15] M. G. Vavilov and L. I. Glazman, Phys. Rev. Lett. 94, 086805 (2005).

[16] W. H. Zurek, Phys. Today 44, 36 (1991).

[17] U. Weiss, Quantum Dissipative Systems, Second Edition (World Scientific, Singapore, 1999).
[18] L. D. Chang and S. Chakravarty, Phys. Rev. B 31, 154 (1985).

[19] Y. C. Chen, J. Stat. Phys. 49, 811 (1987).

[20] Y. C. Chen, J. Stat. Phys. 47, 17 (1987).

[21] Y. C. Chen, Phys. Rev. A 37, 3450 (1988).

[22] H. Schoeller and G. Schon, Phys. Rev. B 50, 18436 (1994).

[23] M. Keil and H. Schoeller, Chem. Phys. 268, 11 (2001).

[24] T. Takagahara, Quantum Coherence, Correlation and Decoherence in Semiconductor Nanostructures (ACADEMIC PRESS, UK, 2003), chap. 9.

[25] Y. Makhlin, G. Schön, and A. Shnirman, Chem. Phys. 296, 315 (2004).

[26] B. Fischer and M. W. Klein, Phys. Rev. B 11, 2025 (1975).

[27] Y. Yafet, Phys. Rev. B 36, 3948 (1987).

[28] V. I. Litvinov and V. K. Dugaev, Phys. Rev. B 58, 3584 (1998).

[29] J. Korringa, Physica. 16, 601 (1950).

[30] D. Goldhaber-Gordon, J. Gores, M. A. Kastner, H. Shtrikman, D. Mahalu, and U. Meirav, Phys. Rev. Lett. 81, 5225 (1998).

[31] A. Kogan, G. Granger, M. A. Kastner, D. GoldhaberGordon, and H. Shtrikman, Phys. Rev. B 67, 113309 (2003).

[32] D. Loss and D. P. DiVincenzo, Phys. Rev. A 57, 120 (1998).

[33] K. Ueno, K. Saiki, and A. Koma, Jpn. J. Appl. Phys. Part 1 - Regul. Pap. Short Notes Rev. Pap. 40, 1888 (2001).

[34] A.A. Shklyaev and M. Ichikawa, Appl. Phys. Lett. 80, 1432 (2001).

[35] A.A. Shklyaev and M. Ichikawa, Phys. Rev. B 65, 045307 (2001). 